\title{
Effect of an intersection of carbon nanotubes on the carrier accumulation under an external electric field
}

Taketo Kochi and Susumu Okada

Graduate School of Pure and Applied Sciences, University of Tsukuba, Tsukuba, Ibaraki 305-8571, Japan

We studied the electronic structure of semiconducting carbon nanotubes (CNTs) thin films, in which CNTs intersect each other, under an external electric field, using first-principles total-energy calculations within the framework of the density functional theory. Our calculations show that distribution of the accumulated carriers strongly depends on CNT species, their mutual arrangement with respect to the electrode, and carrier concentrations. Under the particular condition, an induced electric field between CNT is opposite to the applied field. We also showed that quantum capacitance of the CNT thin films also depend on relative arrangement of CNT to the electrode.

Carbon nanotubes (CNTs) are attracting much attention owing to their unique geometric and electronic properties. ${ }^{1-8)}$ In particular, because CNTs have tubular structures with a nanometer diameter, CNTs are regarded as emerging materials for advancing further miniaturization of the electronic devices that is one of important demands in the semiconductor device technology. Indeed, it has been demonstrated that an individual CNT works as a conducting channel of field effect transistors (FETs). ${ }^{9-12)}$ In addition, mat films of CNTs also work as conducting channels for the electronic devices. ${ }^{13,14)}$ In these typical CNT-based electronic devices, CNTs intrinsically interact with other CNTs and attach to foreign materials, leading to the structural deformations or defects those seriously modulate their electronic properties. ${ }^{15-20)}$ In our previous works, defects and structural deformation affect the carrier accumulation in CNT under the FET structure. ${ }^{21-24)}$ On the other hand, although the experiments showed that mat films of CNT can work as conducting channels in real devices, it is still unclear a microscopic mechanism of the carrier accumulation in CNTs thin films, in which CNTs possess intersections with other CNTs, by an external electric field.

In this manuscript, we aim to elucidate the influence of the intersection of CNTs on the carrier accumulation under an external electric field using first-principles total energy calculations within the framework of the density functional theory. Our calculations showed that the carrier accumulation into CNT thin films with the intersections due to the electric field depends on the field strength, CNT species, and their arrangement to the electrode. For most of thin films consisting of $(10,0)$ and $(11,0) \mathrm{CNTs}$, the accumulated carriers are primary distributed on the wall of CNT facing to the counter electrode. However, for the thin films in 
which $(10,0)$ CNT is located at the electrode side, the accumulated carriers are spilled out the other CNT situated at the second subsurface of the thin films, leading to an unusual electric field of which direction is opposite to the applied field.

All calculations are performed based on the density functional theory ${ }^{25,26)}$ using a STATE package. ${ }^{27}$ ) We use local density approximation (LDA) to express the exchange-correlation energy among interacting electrons. ${ }^{28,29)}$ An ultrasoft pseudopotential is adopted for describing the interaction between nuclei and electrons. ${ }^{30}$ ) The valence wave functions and charge density were expanded with a plane-wave basis set with cutoff energies of 25 and 225 Ry, respectively. We adopted the effective screening medium (ESM) method $^{31)}$ to solve the Poisson equation including excess electrons or holes injected by the electrode within the framework of the DFT using the plane-wave basis set. To simulate the CNT thin films in the top-gate FET structure, we consider the structural model shown in Fig. 1: The thin films are simulated by a CNT bilayer consisting of $(10,0)$ and $(11,0)$ CNTs, which intersect each other with angles of 60,75 , and 90 degrees, located below the planar gate electrode simulated by an effective screening medium with vacuum spacing of 12.48-12.67 $\AA$ and 11.16-11.35 $\AA$ depending on the intersectional angle for the cases that $(10,0)$ and $(11,0)$ CNTs are located at the electrode side, respectively. To simulate the CNT bilayer with the above intersectional angles, we consider quadruple periodicity of zigzag CNTs with parallelogram cells in which the CNT are separated by 6.92-9.20 $\AA$-spacing along lateral directions, depending on the CNT species and intersectional angles. Each CNT is optimized in its isolated form until the force acting on each atom was less than $5 \mathrm{mRy} / \AA$. During the calculations on the thin films with and without electric field, internal atomic coordinate of each CNT is fixed to that in its isolated form, because the force acting on each atom in the FET structure is still smaller than the force cutoff criterion.

Figure 2 shows the intertube interaction between two CNTs with intersectional angles of 60,75 , and 90 degrees as a function of the intertube distance. The intertube interaction is evaluated as

$$
E=E_{\mathrm{CNT} 1+\mathrm{CNT} 2}-E_{\mathrm{CNT} 1}-E_{\mathrm{CNT} 2}
$$

where $E_{\mathrm{CNT} 1+\mathrm{CNT} 2}, E_{\mathrm{CNT} 1}$, and $E_{\mathrm{CNT} 2}$ denote the total energies of the CNT thin film, CNT1, and CNT2, respectively. The optimum interwall spacing between CNTs is sensitive to CNT species and their mutual stacking angle. The optimum CNT spacing is 2.9, 3.0, and 3.1 $\AA$ for intersectional angles of 60, 75, and 90 degree, respectively, for the thin films consisting of $(10,0)$ CNTs. For the thin films consisting of $(10,0)$ and $(11,0)$, the optimum spacing is 3.1 $\AA$, irrespective to the intersectional angles studied here. For the thin film consisting of $(11,0)$ CNTs, the optimum spacing is 3.0, 3.1, and $3.1 \AA$ for intersectional angles of 60,75 , and 90 degree, respectively. The intertube interaction under the angle of 60 degree is the largest, because of the large contact area under the shallow intersectional angle. In addition to the contact area, relative atomic arrangements between CNTs at the contact region also cause the 


\section{(a)}

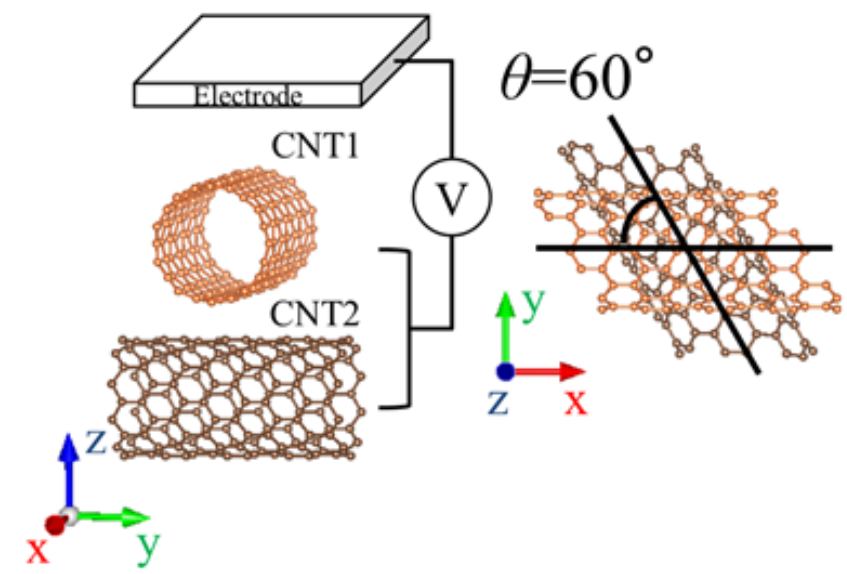

(c)
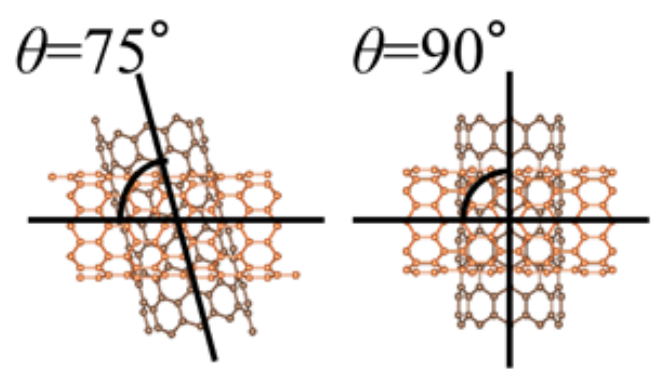

Fig. 1. (a) Schematic view of the structural model of CNT thin films, CNT1-CNT2, in the FET structure under the electric field. CNT1 and CNT2 denote the CNT situated at electrode and opposite sides, respectively. The top views of CNT thin films with the intersectional angles of (b) $\theta=60$, (c) 75 , and (d) 90 degrees.

(a)

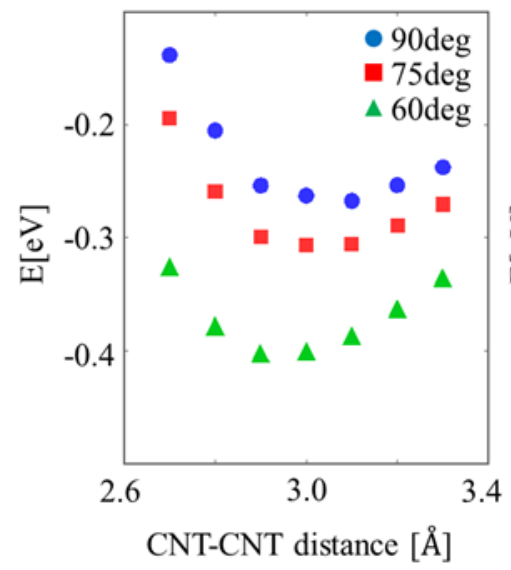

(b)

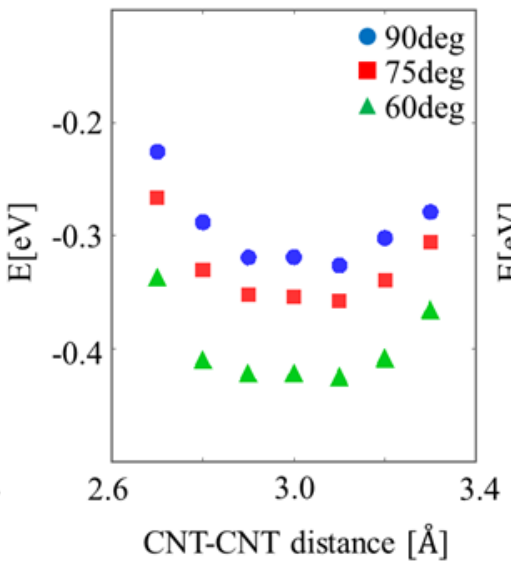

(c)

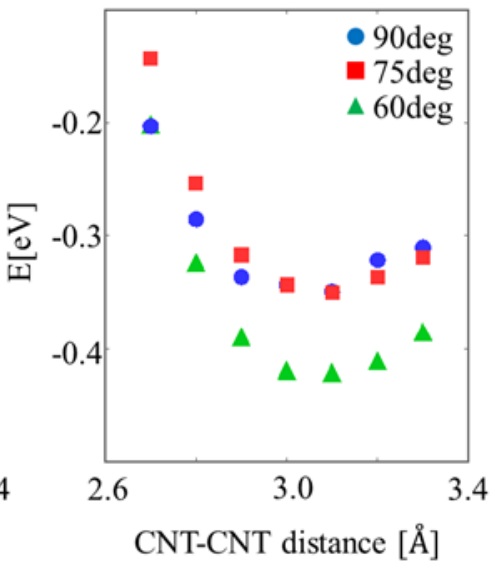

Fig. 2. The intertube interaction in CNT thin films of (a) $(10,0)-(10,0),(b)(10,0)-(11,0)$, and (c) $(11,0)-(11,0)$ CNT as a function of the intertube distance. Triangles, squares, and circles denote the intertube interaction of CNTs with intersectional angles of 60,75 , and 90 degrees, respectively.

binding energy difference. For all CNT thin films with the intersectional angle of 60 degree, atoms at the interfaces are similar arrangement to the AB-stacking of graphite. Thus, the relative atomic arrangements also play important roles to determine the energetics.

Figure 3 shows the distribution of accumulated carriers in the CNT thin films under an electric field. In all cases, the accumulated electrons and holes are mainly distributed on CNT situated at the electrode side. However, the accumulated carriers are slightly spilled over the 
second subsurface CNT opposite to the electrode for the thin films in which $(10,0)$ CNT is located at the electrode side under electron and hole doping. By focusing on the detailed carrier density induced by the electric field, the carrier distribution depends on the carrier species. For the electron doping in the thin film in which the $(10,0) \mathrm{CNT}$ is located at the electrode side, electrons are induced on the upper part (electrode side) of (10,0) CNT, while the holes are induced on the lower part (opposite side) of the $(10,0) \mathrm{CNT}$, leading to the dipole moment in the $(10,0)$ CNT. In accordance with the induced carrier, the electrons are accumulated in the top of the second subsurface CNT. In contrast, for the hole doping in all CNT configurations, only holes are induced throughout the wall of the CNT situated at the electrode side. On the second subsurface CNT, holes are slightly induced under the CNT conformation in which the $(10,0)$ CNT is located at electrode side, while the electrons are induced in the second subsurface CNT for the $(11,0)-(10,0)$ thin film with the intersectional angles of 75 and 90 degrees. Thus, the distribution of accumulated carriers depends on the intersectional angle, CNT species, and the distance between two CNTs.

Figure 4 shows contour plots of the electrostatic potential and vector plots of the electric field of the CNT thin films under an electric field. In all cases, the electric field is well screened in the first CNT layer irrespective to the CNT species and their conformations, reflecting that the electric field is mainly concentrated at the upper part of the CNT situated at the electrode side. Note that the field concentration depends on the carrier species. Because of the characteristic carrier distribution in the thin films with $(10,0)-(n, 0)$ thin film under the electron doping, we find that the substantial electric field in the space between CNTs due to the induced holes and electrons on $(10,0)$ and $(n, 0)$ CNTs, respectively.

By focusing on the electric field between CNTs, we find that the electric field between CNTs is sensitve to the CNT arrangements and carrier species. For the electron doping, the induced electric field between CNTs in the thin film of $(10,0)-(10,0)$ CNT with the angle of 60 degree exhbit unusual feature. We found the weak induced electric filed of which direction is opposite to the applyied electric field [Fig. 5(a)]. In contrast, the induced field in the thin films of $(10,0)-(10,0)$ with other angles is the same direction to the external field. Furthermore, the other thin films, i,e, $(10,0)-(11,0),(11,0)-(10,0)$, and $(11,0)-(11,0)$, also do not exhibit such unusual field profile.

As shown in Fig. 5 (c), field direction between CNTs is opposite to that of the external field in the (11,0)-(10,0) thin films with the intersectional angles of 75 and 90 degrees. This unusual electric field is ascribed to the distribution of accumulated carriers on the second subsurface CNT. In the (11,0)-(10,0) thin film with intersectional angles of 75 and 90 degrees, the electrons are induced in the second subsurface $(10,0)$ CNT, leading to the electric field of which direction is opposite to the external electric field. In contrast, we could not find such unusual induced electric field between CNT in the $(10,0)-(11,0)$ thin film under hole doping 
(a)

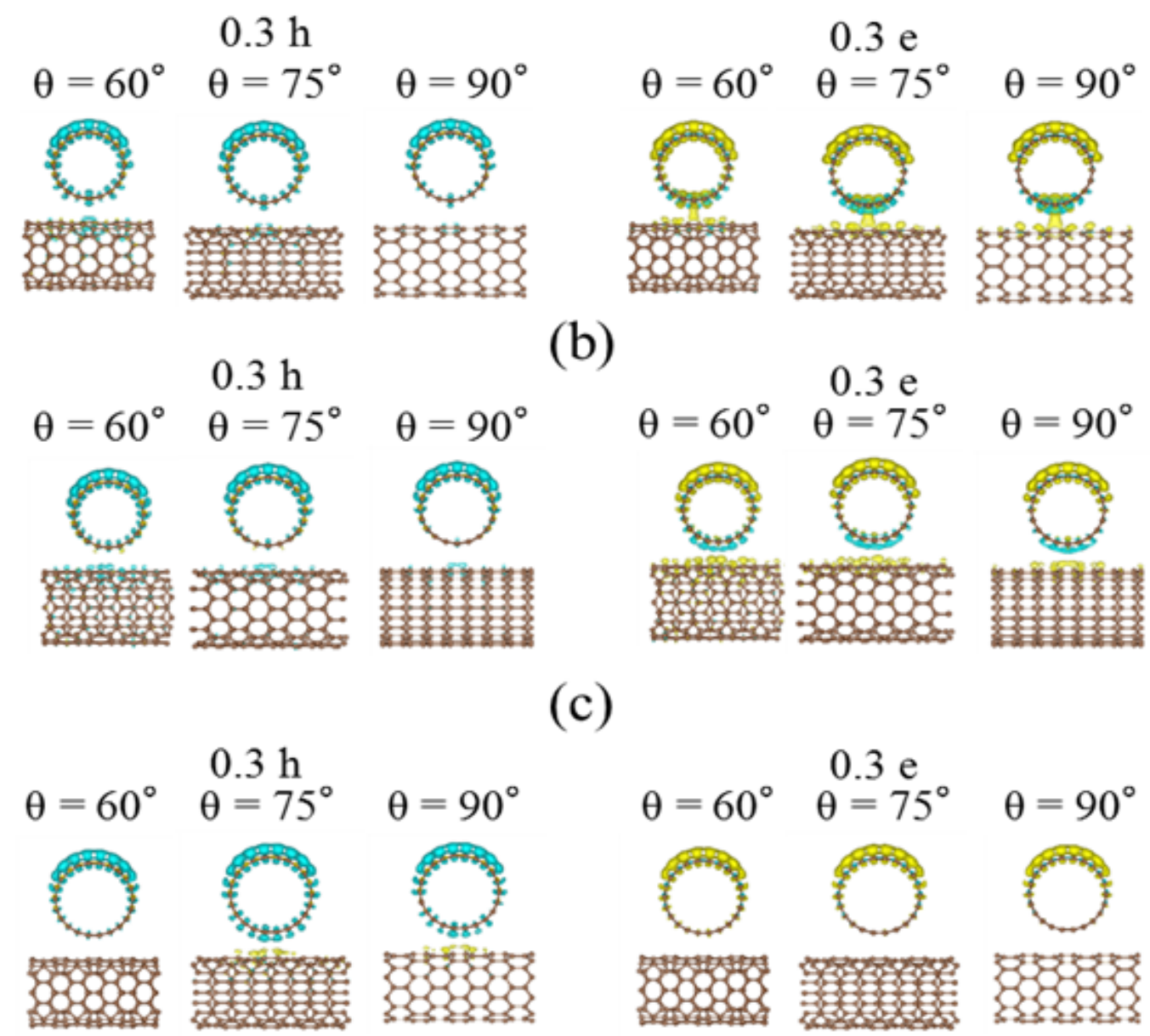

(d)

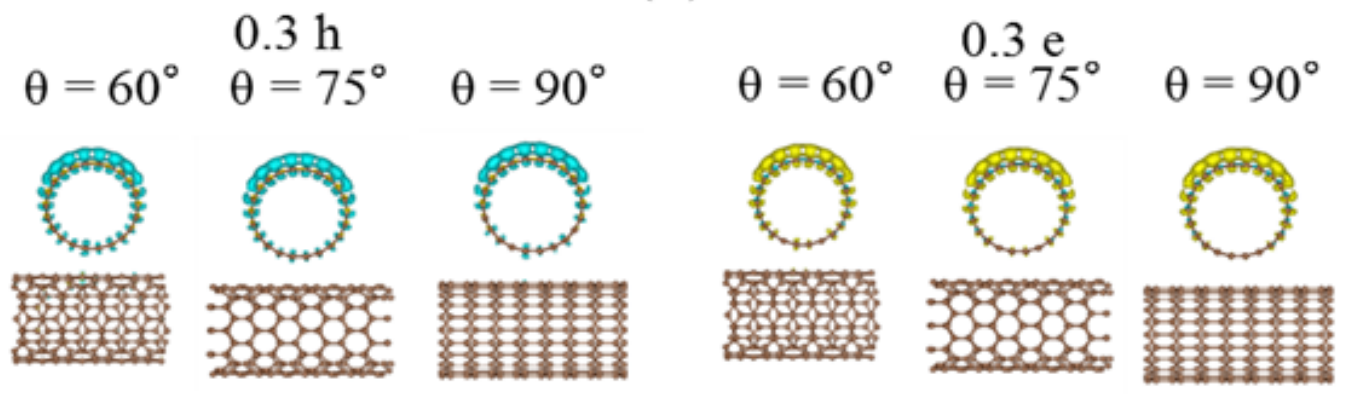

Fig. 3. Isosurfaces of the accumulated carriers in CNT thin films of (a) $(10,0)-(10,0),(b)(10,0)$ $(11,0),(\mathrm{c})(11,0)-(10,0)$, and (d) (11,0)-(11,0) CNTs. In each panel, 0.3h and 0.3e denote the injection of 0.3 holes and 0.3 electrons, respectively. Yellow and blue isosurfaces indicate regions where electrons and holes increase, respectively, under the electric field. 
[Fig. 5(d)]. This is because that the holes are induced in the wall of the second subsurface CNT. The facts imply that the work function difference of CNT causes the unusual electric field between CNT arising from the distribution of the accumulated carriers.

Figure 6 shows the total capacitance of the CNT thin films as a function of the gate voltages for the electron and hole injection. Note that the total capacitance is defined as

$$
C=\frac{d Q}{d \mu}=\frac{C_{\text {cla }} D}{C_{\text {cla }}+D},
$$

where $C_{\text {cla }}$ and $D$ denote the geometrical capacitance and density of states of CNT, respectively. ${ }^{32,33)}$ The capacitance of CNTs depends on the CNT species, their mutual arrangements, and the gate voltage. By applying the gate voltage, the capacitance rapidly increases by reflecting the first van Hove singularity depending on the CNT arrangements. Then with further increase of the voltage, the capacitance weakly depend on the gate voltage, because of the gradually decreasing density of states in both conduction and valence bands. It is assumed that the density of states of the CNTs cause variation in the capacitance as the bias voltage. In addition, from the viewpoint of the intersectional angle, the capacitance increases with increasing the intersectional angles irrespective to the CNT species (Fig. 6). Thus, the capacitance depends on constituent CNT species and their intersectional morphology.

Acknowledgements This work was supported in part by a KAKENHI, MEXT and a ZE-program, Institute of Advanced Energy, Kyoto University. Computations were performed on a NEC SX-8/4B at the University of Tsukuba, a SGI ICE XA/UV at the Institute for Solid State Physics, The University of Tokyo, and a NEC SX-Ace at the Cybermedia Center, Osaka University. 
$0.3 \mathrm{~h}$

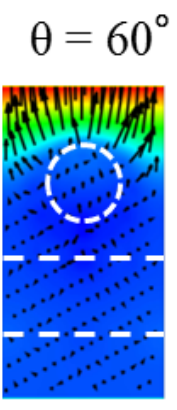

$0.3 \mathrm{~h}$

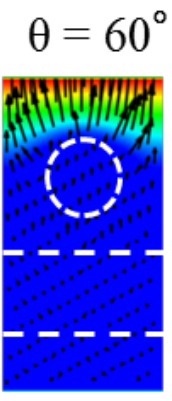

$0.3 \mathrm{~h}$

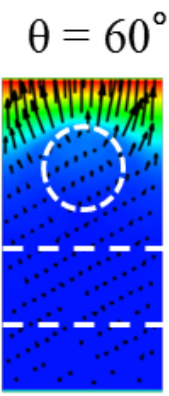

$0.3 \mathrm{~h}$

$\theta=60^{\circ}$

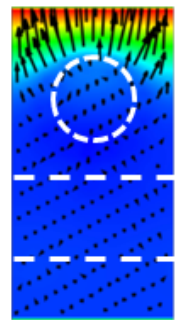

(a)

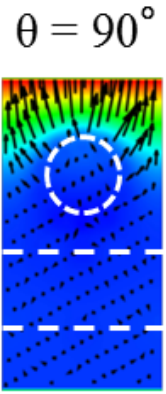

$\theta=60^{\circ}$
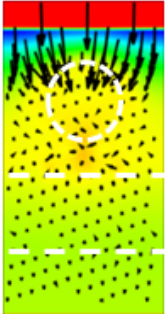

(b)

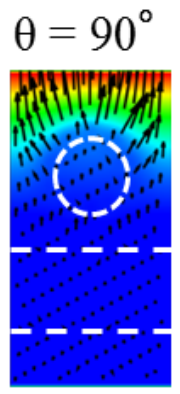

$\theta=60^{\circ}$
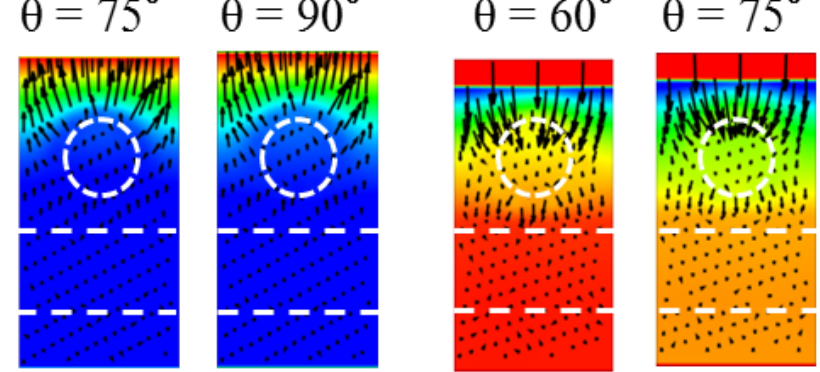

(c)

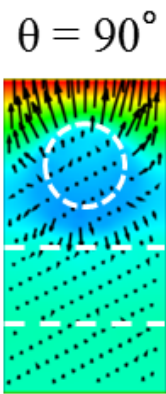

$0.3 \mathrm{e}$

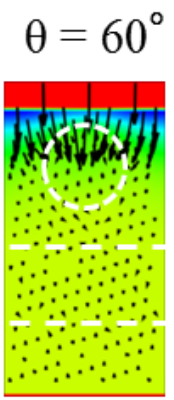

$\theta=75^{\circ}$

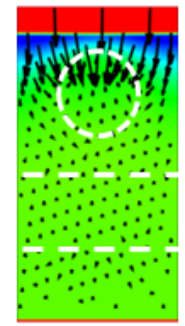

(d)

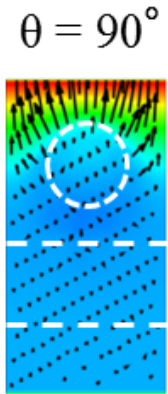

$0.3 \mathrm{e}$

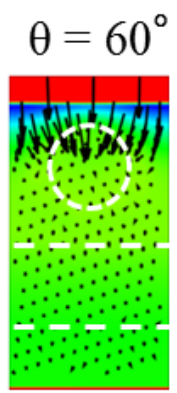

$\theta=90^{\circ}$

$\theta=90^{\circ}$
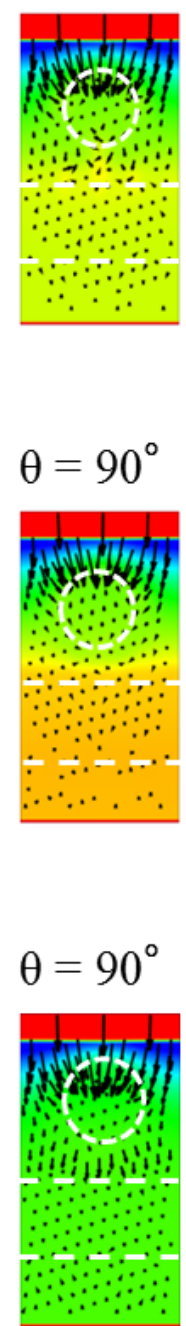

....... $\theta=90^{\circ}$
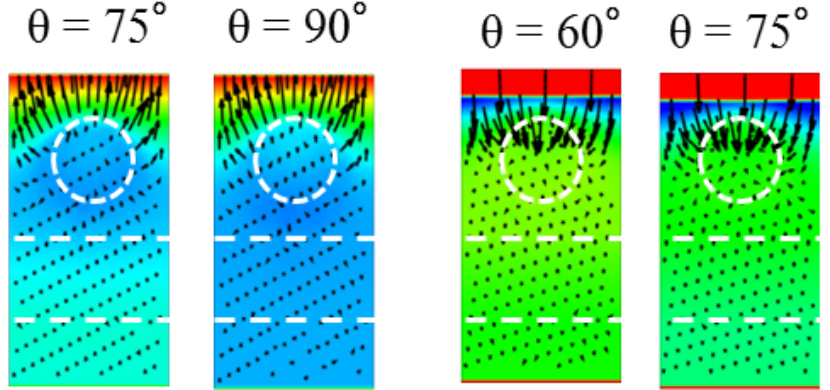

$\theta=90^{\circ}$

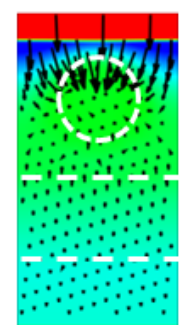

Fig. 4. Counter plots of the electrostatic potential and vector plots of the electric field of CNT thin films of (a) (10,0)-(10,0), (b) (10,0)-(11,0), (c) (11,0)-(10,0), and (d) (11,0)-(11,0) CNTs. In each panel, $0.3 \mathrm{~h}$ and $0.3 \mathrm{e}$ denote the injection of 0.3 holes and 0.3 electrons, respectively. The white dotted lines in each panel indicate the walls of CNTs. 
(a)

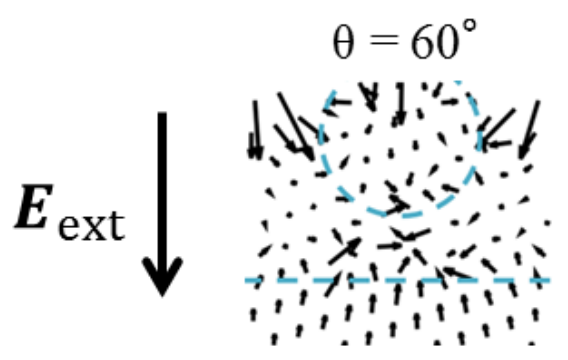

$0.3 \mathrm{e}$

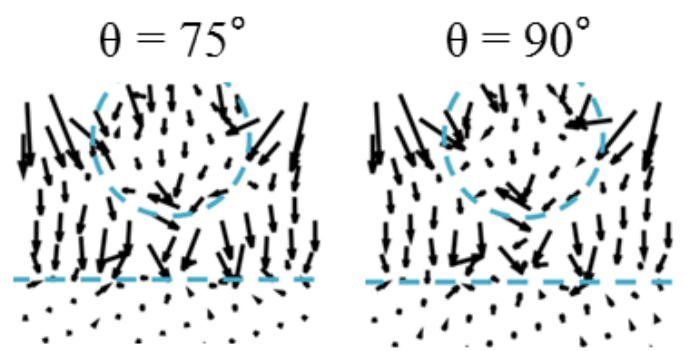

(b)

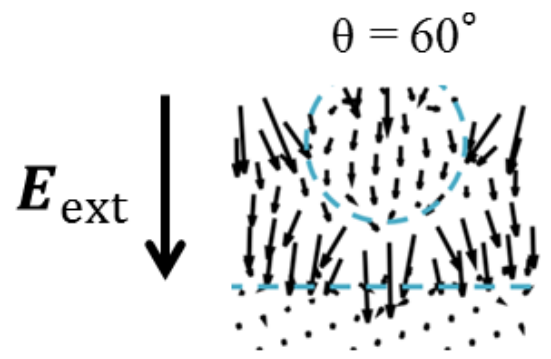

$0.3 \mathrm{e}$
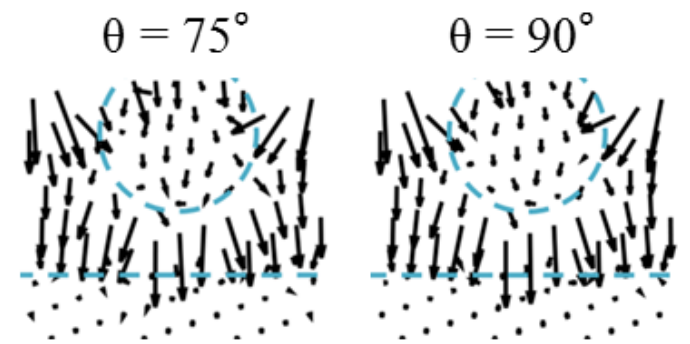

(c)

$0.3 \mathrm{~h}$

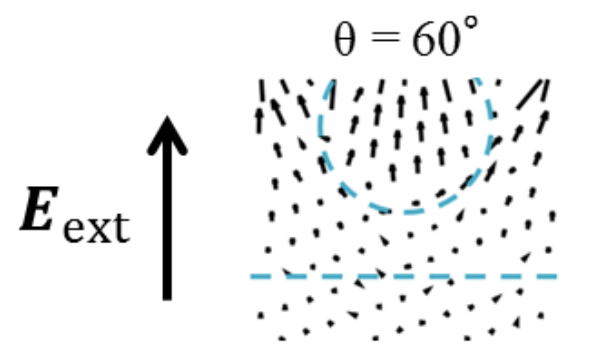

$\theta=75^{\circ}$

$$
\theta=90^{\circ}
$$
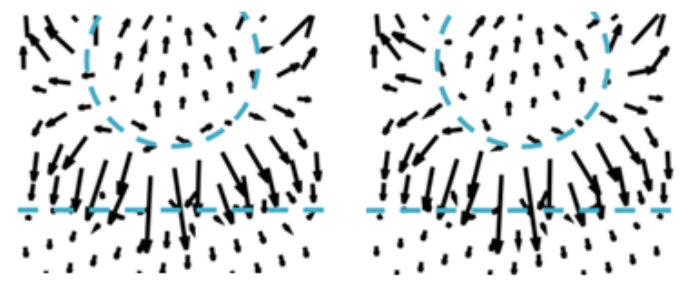

(d)

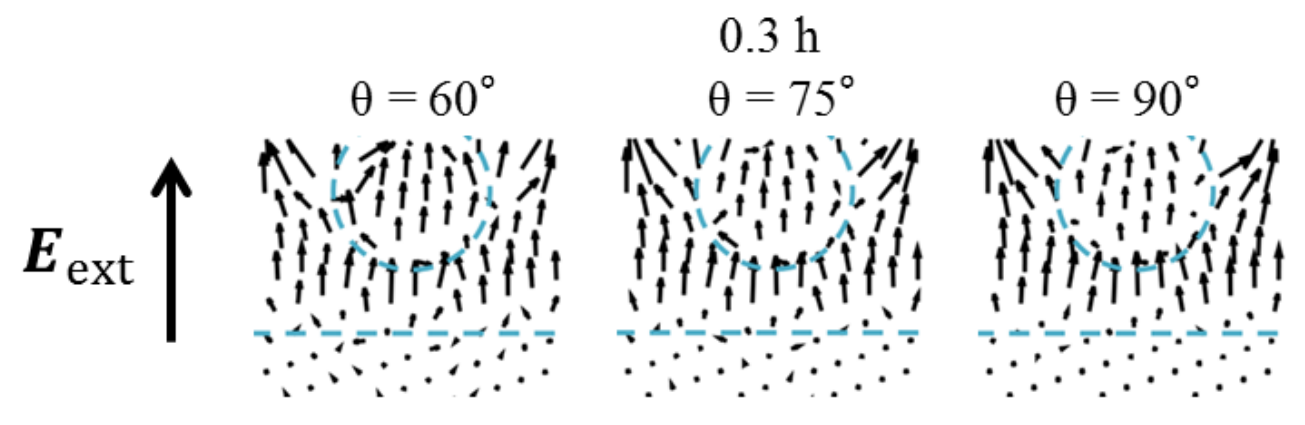

Fig. 5. Enlarged images of the electric field at CNT interfaces of CNT thin films of (a) $(10,0)-(10,0)$, (b) $(10,0)-(11,0),($ c) $(11,0)-(10,0)$, and (d) $(10,0)-(11,0)$ CNTs. In each panel, 0.3h and 0.3e denote the injection of 0.3 holes and 0.3 electrons, respectively. The blue dotted lines in each panel indicate the walls of CNTs. Arrows outside the panals indicate the direction of external electric field $\boldsymbol{E}_{\text {ext }}$. 
(a)

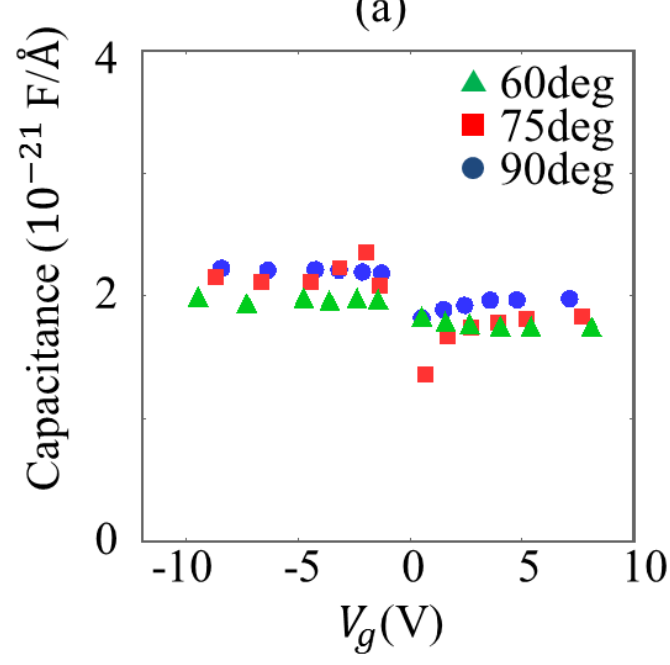

(c)

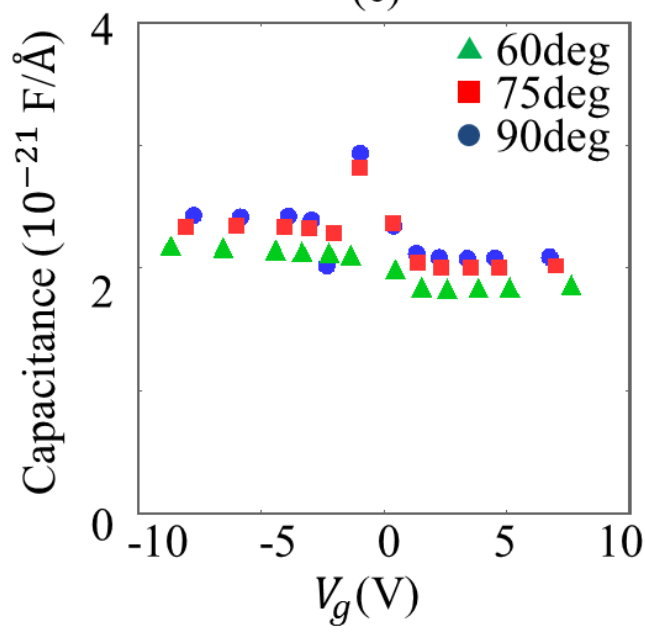

(b)

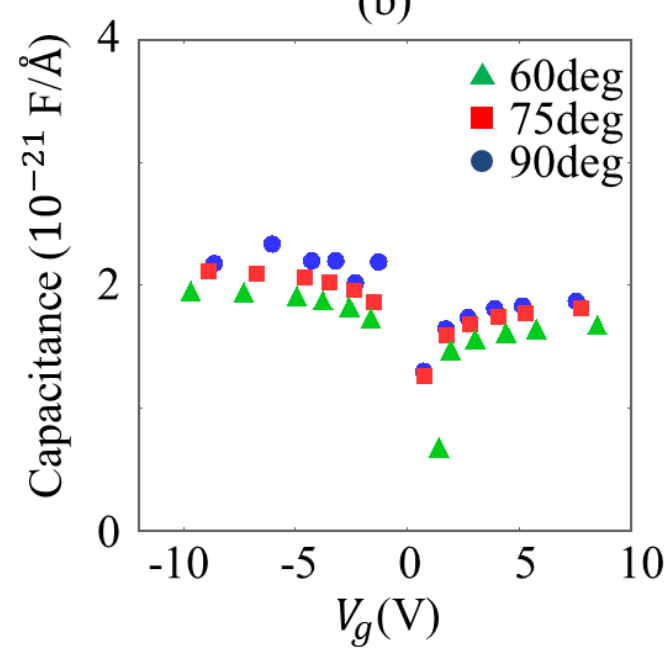

(d)

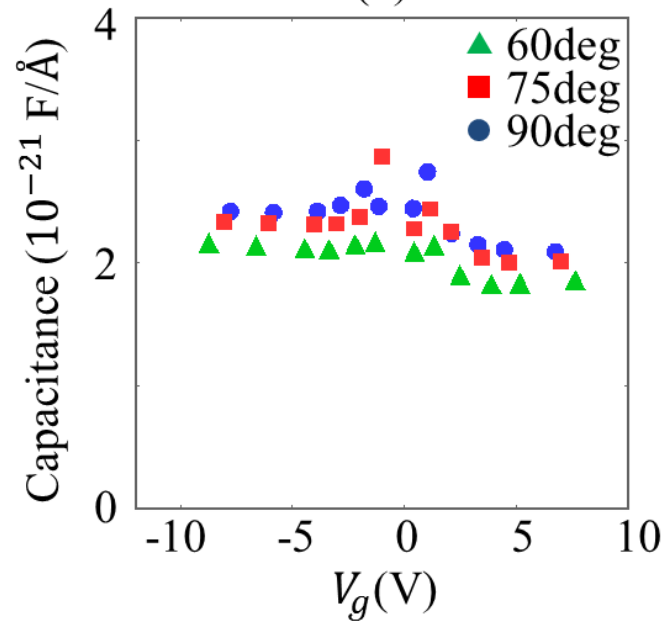

Fig. 6. Capacitances of (a) (10,0)-(10,0), (b) (10,0)-(11,0), (c) $(11,0)-(10,0)$, and (d) $(11,0)-(11,0)$ thin films as a function of the gate voltages for electron or hole injections. Triangles, circles and squares denote the capacitance for intersectional angles of 60, 75, and 90 degree, respectively. 


\section{References}

1) S. Iijima, Nature 354, 56 (1991).

2) N. Hamada, S. Sawada, and A. Oshiyama, Phys. Rev. Lett. 68, 1579 (1992).

3) R. Saito, M. Fujita, G. Dresselhaus, and M. S. Dresselhaus, Appl. Phys. Lett. 60, 2204 (1992).

4) K. Tanaka, K. Okahara, M. Okada, and T. Yamabe, Chem. Phys. Lett. 191, 469 (1992).

5) R. S. Ruoff and D. C. Lorents, Carbon 33, 925 (1995).

6) M. M. J. Treacy, T. W. Ebbesen, and J. M. Gibson, Nature 381, 678 (1996).

7) S. Berber, Y.-K. Kwon, and D. Tománek, Phys. Rev. Lett. 84, 4613 (2000).

8) T. Yamamoto, S. Watanabe, and K. Watanabe, Phys. Rev. Lett. 92, 075502 (2004).

9) S. J. Tans, A. R. M. Verschueren, and C. Dekker, Nature 393, 49 (1998).

10) R. Martel, T. Schmidt, H. R. Shea, T. Hartel, and Ph. Avouris, Appl. Phys. Lett. 73, 2447 (1998).

11) Y. Nosho, Y. Ohno, S. Kishimoto, and T. Mizutani, Appl. Phys. Lett. 86, 073105 (2005).

12) Y. Nosho, Y. Ohno, S. Kishimoto, and T. Mizutani, Nanotechnology 17, 3412 (2006).

13) D.-M. Sun, M. Y. Timmermans, Y. Tian, A. G. Nasibulin, E. I. Kauppinen, S. Kishimoto, T. Mizutani, and Y. Ohno, Nat. Nanotechnol. 6, 156 (2011).

14) D.-M. Sun, M. Y. Timmermans, A. Kaskela, A. G. Nasibulin, S. Kishimoto,T. Mizutani, E. I. Kauppinen, and Y. Ohno, Nat. Commun. 4, 2302 (2013).

15) M. Igami, T. Nakanishi, and T. Ando, J. Phys. Soc. Jpn. 68, 716 (1999).

16) P. O. Lehtinen, A. S. Foster, Y. Ma, A. V. Krasheninnikov, and R. M. Nieminen, Phys. Rev. Lett. 93, 187202 (2004).

17) Y. Ma, P. O. Lehtinen, A. S. Foster, and R. M. Nieminen, New J. Phys. 6, 68 (2004).

18) S. Okada, K. Nakada, K. Kuwabara, K. Daigoku, and T.Kawai, Phys. Rev. B 74, 121412(R) (2006).

19) N. G. Chopra, L. X. Benedict, V. H. Crespi, M. L. Cohen, S. G. Louie, and A. Zettel, Nature 377, 135 (1995).

20) M. S. C. Mazzoni and H. Chacham, Appl. Phys. Lett. 76, 1561 (2000).

21) U. Ishiyama, N.-T. Cuong, and S. Okada, Jpn. J. Appl. Phys. 53, 115102(2014).

22) U. Ishiyama, N.-T. Cuong, and S. Okada, Jpn. J. Appl. Phys. 54, 065101(2015).

23) U. Ishiyama, N.-T. Cuong, and S. Okada, Appl. Phys. Express 9, 045101(2016).

24) A. Hasegawa and S. Okada, Jpn. J. Appl. Phys. 55, 045101(2016).

25) P. Hohenberg and W. Kohn, Phys. Rev. 136, B864 (1964). 
26) W. Kohn and L. J. Sham, Phys. Rev. 140, A1133 (1965).

27) Y. Morikawa, K. Iwata, and K. Terakura, Appl. Surf. Sci. 169-170, 11 (2001).

28) J. P. Perdew and A. Zunger, Phys. Rev. B 23, 5048 (1981).

29) D. M. Ceperley and B. J. Alder, Phys. Rev. Lett. 45, 566 (1980).

30) D. Vanderbilt, Phys. Rev. B 41, 7892 (1990).

31) M. Otani and O.sugino, Phys. Rev. B 73, 115407 (2006).

32) K. Uchida and S. Okada, Phys. Rev. B 76, 155436 (2007).

33) M. Büttiker, J. Phys.: Condens. Matter 5, 9361 (1993). 